\title{
Survival following Multimodality Treatment Including Surgery for Stage IA-IIIB Small-Cell Lung Cancer
}

\author{
Barbara Christine Weckler ${ }^{1}$ Natalie Baldes ${ }^{1}$ Joachim Schirren ${ }^{1}$ \\ ${ }^{1}$ Department of Thoracic Surgery, HELIOS Dr. Horst Schmidt Kliniken \\ Wiesbaden, Wiesbaden, Hessen, Germany \\ Thorac Cardiovasc Surg 2019;67:291-298. \\ Address for correspondence Barbara Christine Weckler, MD, \\ Department of Thoracic Surgery, HELIOS Dr. Horst Schmidt Kliniken \\ Wiesbaden, Ludwig-Erhard-Strasse 100, Wiesbaden, Hessen 65199, \\ Germany (e-mail: barbara.weckler@gmx.de).
}

\begin{abstract}
Background Prognosis in limited disease small-cell lung cancer (SCLC) after concurrent chemoradiotherapy is poor. While some studies show better survival after multimodality treatment including surgery, other trials failed to prove a surgeryrelated survival benefit. Therefore, this study investigated survival in stage IA-IIIB SCLC following surgery combined with chemotherapy and/or thoracic radiotherapy.

Methods We retrospectively reviewed all stage IA-IIIB SCLC patients without supraclavicular lymph node involvement at a single institution between January 1999 and August 2016 after multimodality treatment with curative intent. This comprised surgery consisting of primary tumor resection and systematic lymph node dissection combined with chemotherapy, chemoradiotherapy, or thoracic radiotherapy. Survival was determined using the Kaplan-Meier method, and differences were compared using log-rank tests. The risk of locoregional relapse was calculated.

Keywords

- limited disease smallcell lung cancer

- multimodality treatment for smallcell lung cancer

- lung cancer treatment (surgery

- medical)

Results A total of 47 patients (29 men, 18 women; mean age: 62 years) were included. Thirty-day mortality was $0 \%$. Overall median survival was 56 months, and 2-, $3-, 5-$, and 10 -year survival rates were $69,54,46$, and $30 \%$, respectively. The only significant prognostic factor $(p=0.006)$ was R0 resection $(n=40)$ increasing median survival to 64 versus 17 months in case of technical inoperability $(n=5)$. The risk of locoregional relapse was $2.5 \%(n=1)$ after R0 resection.

Conclusions Multimodality treatment including surgery was safe and led to considerable survival. R0 resection was the only factor extending survival. It could be achieved in most patients and was associated with a low risk of locoregional relapse. Prospective randomized controlled studies are needed to define best practice in stage IA-IIIB SCLC.
\end{abstract}

\section{Introduction}

The current standard of care for stage IA-IIIB small-cell lung cancer (SCLC) consists of four to six cycles of combination chemotherapy, typically cisplatin and etoposide, along with thoracic radiotherapy during the early part of the chemotherapy. This treatment has been shown to result in a median survival of 19 to 23 months and a 5 -year survival of 16 to $26 \%{ }^{1}$

received

December 19, 2017

accepted after revision

June 11, 2018

published online

August 9, 2018

Controversy exists, however, regarding the role of surgery as part of multimodality treatment. While Lad et $\mathrm{al}^{2}$ failed to demonstrate that adding surgery to chemoradiotherapy prolongs survival in stage IA-IIIB SCLC, other studies ${ }^{3-9}$ showed improved survival associated with surgery and chemotherapy with or without thoracic radiotherapy in stage IA-IIIB SCLC including T4 and N3 tumors.

(c) 2019 Georg Thieme Verlag KG Stuttgart . New York
DOI https://doi.org/ 10.1055/s-0038-1667145. ISSN 0171-6425. 
The objective of our study was to investigate survival in stage IA-IIIB SCLC following the combination of surgery, chemotherapy, and thoracic radiotherapy to examine the significance of surgery.

\section{Materials and Methods}

The study protocol had approval by the local ethics committee. This study was conducted according to the revised Declaration of Helsinki and requirements of good clinical practice.

\section{Patient Selection}

All patients with histologically proven stage IA-IIIB SCLC without ipsi- or contralateral supraclavicular lymph node involvement were analyzed retrospectively at a single institution between January 1999 and August 2016. Patients were eligible if they underwent multimodality treatment. This included surgery consisting of primary tumor resection and systematic interlobar, hilar, and mediastinal lymph node dissection combined with chemotherapy, chemoradiotherapy, or thoracic radiotherapy. An interdisciplinary tumor board of specialists in thoracic surgery, oncology, pulmonology, radiotherapy, and radiology determined the individual indication for multimodality treatment including surgery. Indications encompassed likelihood of complete resection of the primary tumor as well as lymph nodes, lack of distant metastases, and adequate performance status along with sufficient cardiopulmonary reserve. Staging procedures comprised computed tomography (CT) of the chest, bronchoscopy, magnetic resonance imaging (MRI) or CT of the brain, $\mathrm{CT}$ and/or ultrasound of the abdomen, and bone scintigraphy. In recent years, endobronchial ultrasound, transbronchial fine needle aspiration, and fludeoxyglucose positron emission tomography-CT (PET-CT) instead of CT of the abdomen and bone scintigraphy were performed in some of the patients. Histology was taken through bronchoscopy, mediastinoscopy, or CT-guided puncture. Cardiopulmonary reserve was assessed by electrocardiogram, whole-body plethysmography, blood gases, echocardiography, and exercise test. Initial tumor stage was based on clinical staging procedures. Only if clinical stage was not known and no neoadjuvant treatment was performed, pathological stage after surgery was considered. Disease stage was evaluated using the seventh edition of the tumor node metastasis classification system of the American Joint Committee on Cancer and the Union for International Cancer Control. ${ }^{10}$

\section{Treatment}

Eligible patients with stage IA-IIIB SCLC were assigned to one of three groups:

- Surgery and chemotherapy.

- Surgery and chemotherapy and thoracic radiotherapy.

- Surgery and thoracic radiotherapy.

\section{Surgery}

All patients underwent both primary tumor resection and systematic lymph node dissection. Resection of the primary tumor consisted of lobectomies with or without sleeve resec- tion, bilobectomies with or without sleeve resection, pneumonectomies, resection of a mediastinal tumor, and segmentectomies/subsegmentectomies in case of limited cardiopulmonary reserve. Lymph node dissection comprised systematic interlobar, hilar, and mediastinal lymph node dissection. Right-sided thoracotomies involved lymph node stations 2R, 2L, 4R, 4L, 7, 8, 9, 10R, 10L, 11, and 12, whereas leftsided thoracotomies included lymph node stations $4 \mathrm{~L}, 5,6,7,8$, 9, 10R, 10L, 11, and $12 .{ }^{11}$ If the surgeon decided after diagnostic thoracotomy that complete resection was not going to be achievable, the remaining tumor was left and the operation was finished. This group of patients was considered technically inoperable. In case of neoadjuvant treatment, restaging was performed before deciding on surgery. Surgery was performed only if there was partial or complete remission of the tumor following neoadjuvant treatment.

\section{Chemotherapy, Chemoradiotherapy, Thoracic}

Radiotherapy, and Prophylactic Cranial Irradiation

In addition to surgery, patients received chemotherapy (neoadjuvant and/or adjuvant) either with or without thoracic radiotherapy (neoadjuvant or adjuvant), or adjuvant thoracic radiotherapy.

Prophylactic cranial irradiation was performed after surgery and chemotherapy, and/or thoracic radiotherapy had been finished.

Follow-up data were obtained from clinical records and telephone interviews with treating general practitioners and/ or specialists in oncology, pulmonology, internal medicine, and radiotherapy. Survival was defined as the time from diagnosis to death from any cause. All patients alive at last follow-up were censored. Mortality considered patients who died within 30 days from surgery, chemotherapy, or thoracic radiotherapy. The percentage of patients with second malignancy in addition to SCLC was recorded. These patients were only included if the second malignancy was in complete remission.

Analysis was performed of the impact on survival of stage (IA-IIIA vs. IIIB), T (T1/2 vs. T3/4) and N status (N0/1 vs. N2/3), treatment modality (surgery/chemotherapy vs. surgery/chemotherapy/thoracic radiotherapy; pneumonectomy vs. surgical procedures other than pneumonectomy), resection status (microscopically complete $=\mathrm{R} 0$ resection; microscopically incomplete $=\mathrm{R} 1$ resection; and technical inoperability), age ( $<65$ vs. $\geq 65$ years), and the presence of a second malignancy (existing vs. nonexisting). The risk of locoregional relapse was also calculated.

\section{Statistics}

Descriptive statistics were used to summarize the data. Mean, median, and confidence interval (CI) were given to describe continuous variables, whereas absolute $(n)$ and relative frequencies (percentage rounded to whole numbers) were used for discrete variables. Survival was estimated using the Kaplan-Meier method. Log-rank tests were conducted to compare survival between groups. $p$-values less than 0.05 were considered statistically significant. Statistical analysis was performed with SPSS 15.0 software (SPSS Inc., Chicago, Illinois, United States). 


\section{Results}

\section{Patient Characteristics}

A total of 47 patients were included. Their mean age was 62 years (range: 41-79 years). Of the patients, 29 (62\%) patients were men, 18 (38\%) patients were women. In regard to histology, 41 (87\%) patients had pure SCLC, 5 (11\%) patients had combined SCLC and large cell neuroendocrine carcinoma, and 1 (2\%) patient had combined SCLC and squamous cell lung cancer. Nine (19\%) patients suffered from a second malignancy.

\section{Initial Tumor Stage}

Details on initial tumor stage are summarized in - Table 1.

\section{Treatment}

Twelve (26\%) patients underwent surgery and chemotherapy (neoadjuvant in three patients, adjuvant in six patients, and both neoadjuvant and adjuvant in three patients). Thirty-four (72\%) patients underwent surgery, chemotherapy (neoadjuvant in 23 patients, adjuvant in 6 patients, and both neoadjuvant and adjuvant in 5 patients), and thoracic radiotherapy (neoadjuvant in 1 patient, adjuvant in 33 patients). One (2\%) patient underwent surgery and adjuvant thoracic radiotherapy.

\section{Surgery and R Status Following Surgery}

In terms of type of primary tumor resection, 17 (36\%) patients underwent lobectomy. Sleeve lobectomy, bilobectomy, and sleeve bilobectomy were performed in four (9\%), three (6\%), and four (9\%) patients, respectively. Nine (19\%) patients underwent pneumonectomy (six on the left, three on the right side). Segmentectomy/subsegmentectomy was executed in four (9\%) patients, and resection of a mediastinal tumor was conducted in one (2\%) patient. The remaining five (11\%) patients were technically inoperable.

Table 1 Initial tumor stage in SCLC patients as per UICC (7th edition)

\begin{tabular}{|l|l|l|}
\hline & $n$ & $\left(^{(\%)^{\mathbf{a}}}\right.$ \\
\hline All patients & 47 & $(100)$ \\
\hline IA-IIIA & 37 & $(79)$ \\
\hline IIIB & 10 & $(21)$ \\
\hline T1 & 7 & $(15)$ \\
\hline T2 & 21 & $(45)$ \\
\hline T3 & 7 & $(15)$ \\
\hline T4 & 6 & $(13)$ \\
\hline Missing & 6 & $(13)$ \\
\hline N0 & 17 & $(36)$ \\
\hline N1 & 8 & $(17)$ \\
\hline N2 & 12 & $(26)$ \\
\hline N3 & 6 & $(13)$ \\
\hline Missing & 4 & $(8)$ \\
\hline
\end{tabular}

Abbreviations: SCLC, small-cell lung cancer; UICC, Union for International Cancer Control.

a Some sections of the table do not add up to $100 \%$ due to rounding.
Regarding R status after surgery, 40 (85\%) patients underwent R0 resection, 2 (4\%) patients underwent R1 resection (after pneumonectomy and lobectomy, respectively), and 5 (11\%) patients were technically inoperable.

\section{Chemotherapy}

Out of all 47 patients, 46 (98\%) patients received chemotherapy. Out of these 46 patients, 26 patients underwent neoadjuvant chemotherapy, 12 patients underwent adjuvant chemotherapy, and 8 patients underwent both neoadjuvant and adjuvant chemotherapy. Regarding neoadjuvant chemotherapy, 22 patients received cisplatin or carboplatin combined with etoposide, 2 patients received doxorubicin/ cyclophosphamide/vincristine, and 2 patients underwent chemotherapy of undocumented type. In terms of adjuvant chemotherapy, seven patients received cisplatin or carboplatin combined with etoposide, one patient received doxorubicin/cyclophosphamide/vincristine, and four patients underwent chemotherapy of undocumented type. In regard to neoadjuvant and adjuvant chemotherapy, four patients received neoadjuvant and adjuvant cisplatin or carboplatin in combination with etoposide, two patients received neoadjuvant cisplatin or carboplatin combined with etoposide and adjuvant chemotherapy with undocumented substances, and two patients received neoadjuvant and adjuvant chemotherapy of undocumented type.

\section{Thoracic Radiotherapy}

Out of all 47 patients, 35 (74\%) patients underwent thoracic radiotherapy. Out of these 35 patients, 33 patients underwent adjuvant thoracic radiotherapy and chemotherapy, 1 patient underwent neoadjuvant chemoradiotherapy, and 1 patient underwent adjuvant thoracic radiotherapy without chemotherapy. The total dose of thoracic radiotherapy administered was 45 Gy in 1 patient, 50 Gy in 3 patients, 50.4 Gy in 8 patients, $59.4 \mathrm{~Gy}$ in 2 patients, and $60 \mathrm{~Gy}$ in 2 patients. In the remaining 19 patients, the dose of thoracic radiotherapy was unknown.

\section{Prophylactic Cranial Irradiation}

Twenty-five (53\% of total) patients underwent prophylactic cranial irradiation. Of these 25 patients, the dose of thoracic radiotherapy was $30 \mathrm{~Gy}$ in 5 patients, $30.4 \mathrm{~Gy}$ in 1 patient, and $30.6 \mathrm{~Gy}$ in 2 patients. No details on the dose were available in the remaining 17 patients. One (2\% of total) patient underwent prophylactic cranial irradiation only in part owing to side effects.

\section{Survival}

Overall mortality after multimodality treatment including surgery, chemotherapy, and thoracic radiotherapy was $0 \%$. Overall median survival in all $47(100 \%)$ patients was 56 months (95\% CI: 19-89 months). The corresponding survival curve is shown in - Fig. 1. Overall 2-, 3-, 5-, and 10 -year survival rates were $69,54,46$, and $30 \%$, respectively.

The only statistically significant prognostic survival factor was $\mathrm{R} 0$ resection, which was associated with increased median survival of 64 versus 17 months in case of technical inoperability $(p=0.006)$. Survival after $\mathrm{R} 1$ resection was 12 and 21 months 


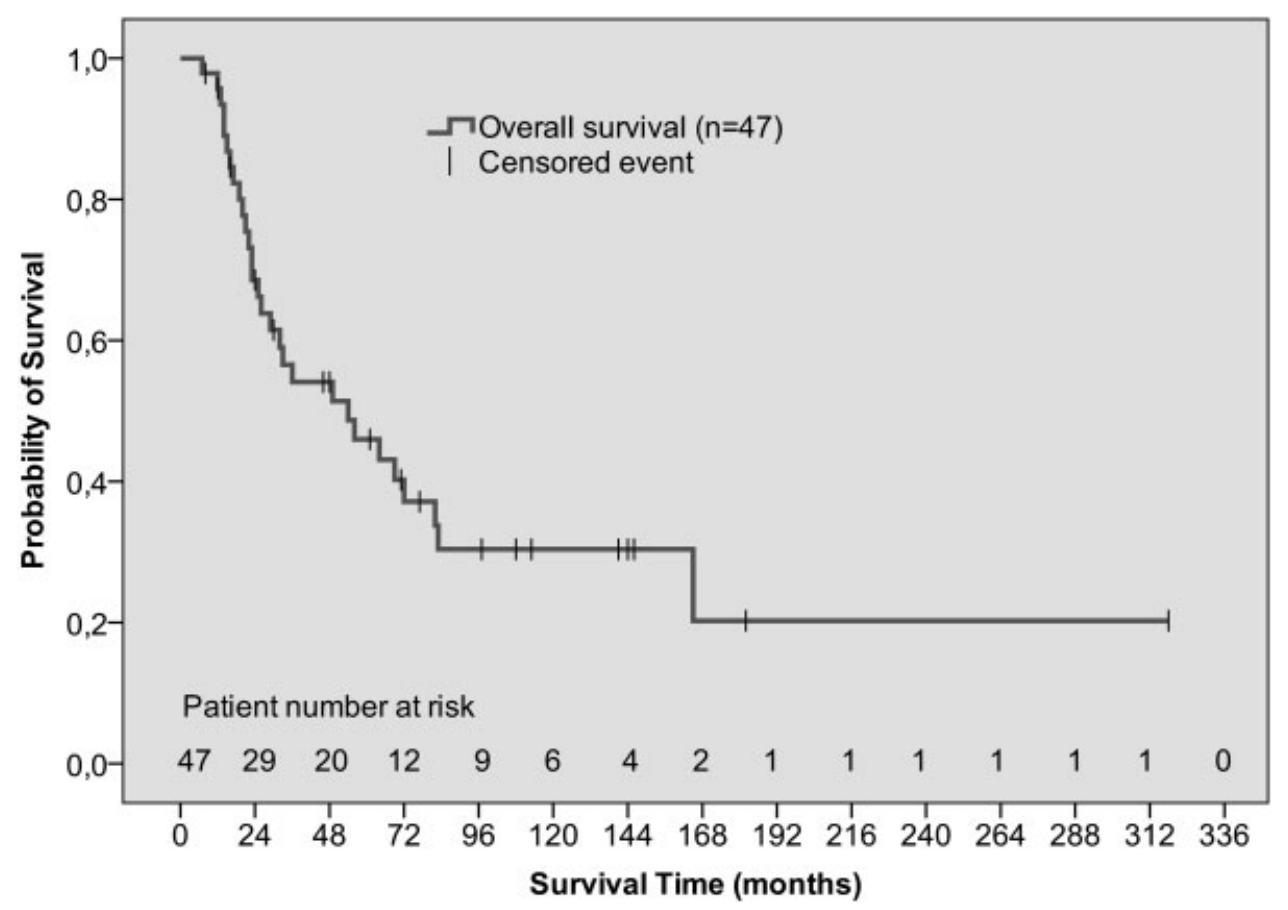

Fig. 1 Overall survival in stage IA-IIIB small-cell lung cancer patients after multimodality treatment including surgery.

$(n=2)$. The corresponding details and survival curves are provided in - Table 2 and - Fig. 2. Stage, T and N status, additional thoracic radiotherapy, pneumonectomy, age, and the presence of a second malignancy did not have a statistically significant impact on median survival (-Table $\mathbf{2}$ ).

\section{Risk of Locoregional Relapse}

Locoregional relapse following multimodality treatment including surgery occurred in 1 (2.5\%) of 40 patients after R0 resection.

\section{Discussion}

The central result of this study was that multimodality treatment including surgery, chemotherapy, and thoracic radiotherapy was safe and led to respectable survival in stage IA-IIIB SCLC. The only significant factor prolonging survival was $\mathrm{R} 0$ resection, which was also associated with a low risk of locoregional failure.

\section{Mortality}

In our study, no patient died from a cause associated with multimodality treatment including surgery. Similarly, Eberhardt et $\mathrm{al}^{4}$ showed that 1 (2\%) of 46 SCLC patients in stage IB-IIIB died of septicemia during the first chemotherapy cycle as part of multimodality treatment including surgery, chemotherapy, and thoracic radiotherapy. No additional treatment-related death was recorded. This indicates a high level of safety of this combination therapy.

\section{Overall Survival}

Our study confirmed an overall median survival of 56 months and a 5-year survival rate of $46 \%$. Comparably, Eberhardt et $\mathrm{al}^{4}$ demonstrated an overall median survival of 36 months and a 5 -year survival of $46 \%$ in stage IB-IIIB SCLC after neoadjuvant chemotherapy or chemoradiotherapy followed by surgery or definitive chemoradiotherapy. Schreiber et $\mathrm{al}^{9}$ showed improved survival after surgery versus nonsurgical treatment in SCLC stage T1-4 and N0-2. Both Zhang et $\mathrm{al}^{5}$ and Lim et al $^{12}$ proved a survival advantage if surgery was part of the treatment for stage I-III SCLC. Welter et $\mathrm{al}^{8}$ reviewed the role of surgery for limited disease SCLC in the literature and concluded that the indication for surgery can be extended to well-selected N2 SCLC patients because of its beneficial impact on survival.

Lad et al, $^{2}$ however, failed to show a survival benefit after surgery was added to chemoradiotherapy in stage IA-IIIB SCLC. The limitations of that study include the choice of the chemotherapy that consisted of cyclophosphamide, doxorubicin, and vincristine, although cisplatin and etoposide were proven to be more effective. ${ }^{13}$ Besides, initial tumor stage was not determined before chemotherapy, and it is unclear whether systematic lymph node dissection was performed. Those limitations might have been decisive for both the limited overall median survival of 16 months and the lack of survival improvement gained from applying surgery in addition to chemoradiotherapy. Consequently, this study ${ }^{2}$ does not seem to provide convincing proof of the advantages of chemoradiotherapy over multimodality treatment including surgery.

Nevertheless, the standard of care for stage IA-IIIB SCLC is concurrent chemoradiotherapy. This treatment is associated with a median survival of 19 to 23 months and a 5-year survival of 16 to $26 \%$, ${ }^{1}$ which appears inferior to the outcome of multimodality treatment including surgery. A prospective study comparing multimodality treatment including surgery 
Table 2 Investigated survival factors in stage IA-IIIB SCLC patients after multimodality treatment including surgery

\begin{tabular}{|c|c|c|c|c|c|}
\hline & $n$ & $(\%)^{a}$ & $\begin{array}{l}\text { Median survival } \\
\text { (months) }\end{array}$ & $\begin{array}{l}95 \% \mathrm{Cl} \\
\text { (months) }\end{array}$ & $p$-Value \\
\hline All patients & 47 & $(100)$ & 56 & $19-89$ & - \\
\hline R0 resection & 40 & (85) & 64 & $39-89$ & \multirow[t]{3}{*}{0.006} \\
\hline Technical inoperability & 5 & $(11)$ & 17 & $15-19$ & \\
\hline R1 resection & 2 & (4) & - & - & \\
\hline UICC stage IA-IIIA & 37 & (79) & 56 & $14-98$ & \multirow[t]{2}{*}{0.774} \\
\hline UICC stage IIIB & 10 & (21) & 49 & $5-93$ & \\
\hline $\mathrm{T} 1 / 2$ & 28 & (60) & 64 & $45-83$ & \multirow[t]{3}{*}{0.918} \\
\hline$T 3 / 4$ & 13 & (28) & 29 & $11-47$ & \\
\hline Missing & 6 & (13) & - & - & \\
\hline $\mathrm{N} 0 / 1$ & 25 & (53) & 64 & $28-100$ & \multirow[t]{3}{*}{0.191} \\
\hline $\mathrm{N} 2 / 3$ & 18 & (38) & 36 & $11-61$ & \\
\hline Missing & 4 & (9) & - & - & \\
\hline Surgery and chemotherapy & 12 & (26) & 72 & $26-118$ & \multirow[t]{3}{*}{0.586} \\
\hline Surgery, chemotherapy, and thoracic RT & 34 & (72) & 36 & $0-74$ & \\
\hline Surgery and thoracic RT & 1 & (2) & - & - & \\
\hline Other than pneumonectomy ${ }^{\mathrm{b}}$ & 38 & $(81)$ & 64 & $21-107$ & \multirow[t]{2}{*}{0.820} \\
\hline Pneumonectomy & 9 & (19) & 49 & $0-116$ & \\
\hline Age $<65$ y & 27 & (57) & 64 & $13-115$ & \multirow[t]{2}{*}{0.283} \\
\hline Age $\geq 65 y$ & 20 & $(43)$ & 36 & $0-94$ & \\
\hline No second malignancy & 38 & (81) & 49 & $20-78$ & \multirow[t]{2}{*}{0.583} \\
\hline Second malignancy & 9 & (19) & 69 & $13-125$ & \\
\hline
\end{tabular}

Abbreviations: Cl, confidence interval; RT, radiotherapy; SCLC, small-cell lung cancer; UICC, Union for International Cancer Control.

a Some sections of the table do not add up to $100 \%$ due to rounding.

${ }^{b}$ Other than pneumonectomy involves lobectomy, sleeve lobectomy, bilobectomy, sleeve bilobectomy, segmentectomy/subsegmentectomy, and resection of a mediastinal tumor.

to concurrent chemoradiotherapy is therefore needed to help define best practice for stage IA-IIIB SCLC.

\section{Importance of RO Resection for Long-Term Survival and Risk of Locoregional Relapse}

The only statistically significant factor extending survival in our study was $\mathrm{R} 0$ resection compared with technical inoperability. Due to the low number of R1 resections ( $n=2)$, they were not compared with $\mathrm{R} 0$ resections nor technical inoperability. Eberhardt et $\mathrm{al}^{4}$ showed a notable increase in median survival to 68 months after $\mathrm{R} 0$ resection combined with neoadjuvant chemotherapy or chemoradiotherapy versus 17 months if R0 resection was not achieved. This supports our finding that $\mathrm{RO}$ resection appears crucial for long-term survival. In our study, surgery consisted of primary tumor resection as well as systematic lymph node dissection. As lymph node metastases in lung cancer are frequent even if clinical staging shows morphologically normal lymph nodes, ${ }^{14}$ systematic lymph node dissection seems mandatory for complete resection in IA-IIIB SCLC in our view.

We demonstrated a low risk of locoregional relapse after R0 resection. Similarly, Eberhardt et $\mathrm{al}^{4}$ proved a risk of locoregional failure of $0 \%$ following $\mathrm{R} 0$ resection combined with either neoadjuvant chemotherapy or chemoradiotherapy in IB-IIIB SCLC. In contrast, Turrisi et $\mathrm{al}^{1}$ showed a risk of locoregional failure of 36 to $52 \%$ after concurrent chemoradiotherapy without surgery. Neither thoracic radiotherapy nor surgery is routinely offered to patients experiencing locoregional failure after chemoradiotherapy. Hence, there are no promising treatment options for these patients. As multimodality treatment including surgery seemed to lower the risk of locoregional failure more effectively, it appears superior to chemoradiotherapy. The reduced risk of locoregional failure after R0 resection could have been decisive for extending survival after $\mathrm{R} 0$ resection in our study. It may also be the main reason why survival in our study seemed improved after multimodality treatment including surgery compared with chemoradiotherapy without surgery as shown by Turrisi et al. ${ }^{1}$

\section{Impact of Tumor Stage as well as $\mathbf{T}$ and $\mathbf{N}$ Status on Survival}

In this study, neither stage nor $\mathrm{T}$ and $\mathrm{N}$ status has a significant impact on survival. Previous trials have not consistently proven that there is a stage limit up to which multimodality treatment including surgery can improve survival. Yang et $\mathrm{al}^{15}$ demonstrated prolonged survival after surgery and 


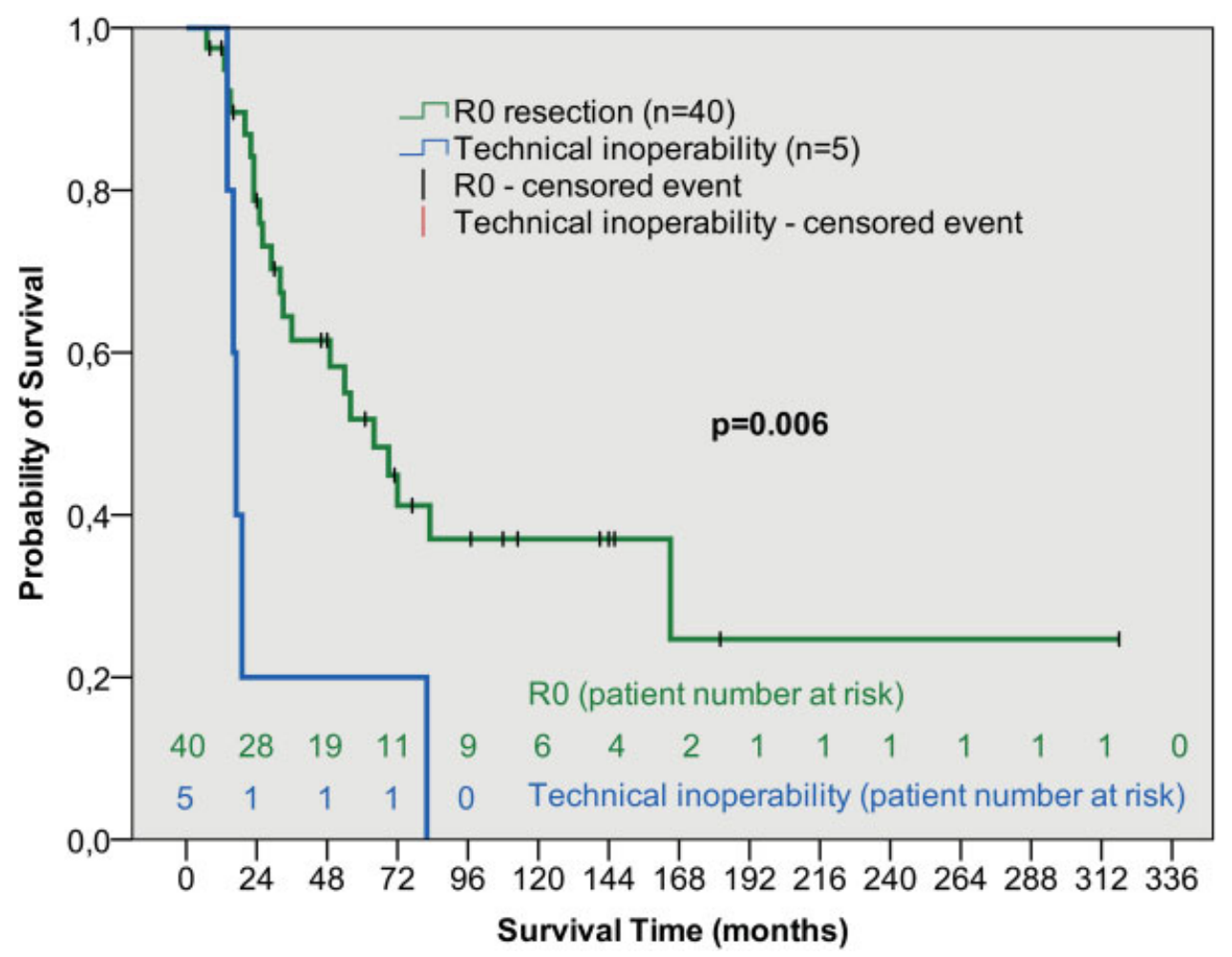

Fig. 2 Survival depending on R0 status versus technical inoperability in stage IA-IIIB small-cell lung cancer patients after multimodality treatment including surgery.

adjuvant chemotherapy with or without thoracic radiotherapy versus concurrent chemoradiotherapy in CT1-3 N1 SCLC. Wakeam et $\mathrm{al}^{6}$ showed extended survival after surgery, chemotherapy, and thoracic radiotherapy versus chemotherapy with or without thoracic radiotherapy in SCLC stage T1-4 NO, any T N1, and any T N2, respectively. Based on a subgroup analysis of stage I/II SCLC, this study also proved significantly better survival after lobectomy and adjuvant chemotherapy versus concurrent chemoradiotherapy. Schreiber et $\mathrm{al}^{9}$ reported improved survival in T1/T2, T3/T4, N0, N1, and N2 SCLC after surgery compared with nonsurgical treatment. In SCLC stage IIIA including N2, median survival was demonstrated to be 46 months after surgery and neoadjuvant chemotherapy. ${ }^{16}$ Zhang et $\mathrm{al}^{5}$ also showed extended survival after surgery and chemotherapy with or without thoracic radiotherapy versus nonsurgical management in SCLC stage IIIA including N2. Thus, several studies ${ }^{5,6,9,16}$ point to a survival advantage associated with surgery and chemotherapy with or without thoracic radiotherapy also in locally/ regionally advanced SCLC.

However, other trials ${ }^{3,17}$ did not show a survival benefit in clinical N2 disease if surgery was added to chemotherapy or chemoradiotherapy. Due to the inconsistent findings, further research is required to determine the role of stage for multimodality treatment including surgery.

Our study included 10 stage IIIB SCLC patients without supraclavicular lymph node involvement. Of these 10 patients, 6 underwent neoadjuvant chemotherapy and adjuvant thoracic radiotherapy, 1 underwent neoadjuvant chemotherapy and adjuvant chemoradiotherapy, 1 underwent neoadjuvant and adjuvant chemotherapy, 1 underwent neoadjuvant chemoradiotherapy without adjuvant treatment, and 1 underwent adjuvant radiotherapy alone for an unknown cause. Median survival was still 49 months in stage IIIB SCLC in our study. This could possibly be attributed to the following reasons:

- Exclusion of patients with supraclavicular lymph node involvement.

- The requirement of partial or complete remission of the tumor after neoadjuvant treatment as a mandatory prerequisite for surgery.

- Strict conduction of complete systematic lymph node dissection as part of each surgery.

Impact of the Sequence of Chemotherapy and Surgery on Survival

Patients receiving neoadjuvant and adjuvant chemotherapy were grouped together since Shepherd et $\mathrm{al}^{7}$ did not demonstrate a survival difference in stage I-IIIA SCLC depending on whether chemotherapy was given prior to or after surgery.

Most of our patients underwent neoadjuvant chemotherapy. The reasons for that were not always recorded in the documents available. In our opinion, there are advantages of neoadjuvant over adjuvant chemotherapy because patients can be selected for surgery depending on tumor response. Only if there is partial or complete remission of the tumor after neoadjuvant chemotherapy, patients seem suitable for surgery. In contrast, if patients receive surgery and adjuvant chemotherapy, there is no information about the response of the tumor to chemotherapy before selecting patients for 
surgery. Nevertheless, more research is required to determine best sequencing of chemotherapy and surgery.

\section{Significance of Thoracic Radiotherapy as Part of Multimodality Treatment}

Our study did not demonstrate a survival benefit after thoracic radiotherapy was added to surgery and chemotherapy. Only one patient underwent surgery and thoracic radiotherapy. The reason why this patient did not receive chemotherapy was not given in the documents available.

Wong et $\mathrm{al}^{18}$ showed an overall detrimental effect of adjuvant radiotherapy on survival in pathological T1-4, NO-2 SCLC patients undergoing complete surgical resection and chemotherapy. When analyzed by subgroup, radiotherapy had an adverse impact on survival particularly in pathological N0 disease. By contrast, radiotherapy was associated with strongly improved survival in pathological N2 disease, whereas it did not significantly influence survival in pathological N1 disease. Schreiber et $\mathrm{al}^{9}$ reported improved survival associated with postoperative radiotherapy in N2 SCLC but not in NO-N1 SCLC.

On the contrary, Wang et $\mathrm{al}^{19}$ showed a trend $(p=0.071)$ toward prolonged survival after adding thoracic radiotherapy to surgery and chemotherapy in stage III SCLC patients. No details were provided on whether systematic lymph node dissection as part of surgery was routinely performed. In contrast, all patients in our study underwent systematic lymph node dissection. The risk of overlooked lymph node metastases was consequently reduced to a minimum so that there was possibly no need for thoracic radiotherapy. In our opinion, systematic lymph node dissection with subsequent complete removal of potential lymph node metastases may be the reason why thoracic radiotherapy did not improve survival in our study.

\section{Impact of Pneumonectomy Compared with Other Types of Resection on Survival}

This study did not prove a significant difference in survival depending on pneumonectomy. In total, nine pneumonectomies were performed. Although preoperative staging took place, it was not clear that pneumonectomy was required for R0 resection in seven patients (five on the left, two on the right side). Pneumonectomy in these cases was conducted because intraoperative staging showed that pneumonectomy was needed for R0 resection. One right-sided pneumonectomy was performed as an emergency procedure in a patient suffering from retention pneumonia as the tumor obstructed the bronchus intermedius and bled. In one patient, the reason for the left-sided pneumonectomy was not documented.

Kawano et $\mathrm{al}^{20}$ demonstrated significantly inferior survival after pneumonectomy versus other surgical procedures in stage I-III SCLC. The conclusion was that pneumonectomy should be avoided. A limitation of this study was that patients did not undergo chemotherapy in addition to surgery. In contrast, improved survival was shown by Yuequan et $\mathrm{al}^{21}$ in stage I-III SCLC if adjuvant chemotherapy was combined with pneumonectomy instead of lobectomy. More research is necessary to investigate whether pneumonectomy combined with chemotherapy and/or thoracic radiotherapy is justifiable if pneumonectomy facilitates $\mathrm{R} 0$ resection.

\section{Study Limitations}

The limitations of this study include the limited number of patients, the lack of a control group treated with concurrent chemoradiotherapy without surgery, and the quality of the data, which depended on exact documentation/interpretation of medical records due to the retrospective design. Survival could also be attributed to patient selection as patients deemed suitable for surgery might have had a better health status than patients receiving chemoradiotherapy without surgery in other studies. Besides, since this study began in 1999, staging procedures have changed toward using PET-CT, endobronchial ultrasound, and transbronchial fine needle aspiration. Therefore, the staging process was not identical in all patients. In addition, our dataset did not provide details on whether patients died of SCLC; therefore, other causes of death cannot be ruled out. Furthermore, patients underwent various types of resection, doses of thoracic radiotherapy, and protocols of chemotherapy. Combination chemotherapy often consisted of substances other than cisplatin and etoposide, although these have been proven to be more effective for SCLC. ${ }^{13}$ Survival might thus have been prolonged if cisplatin and etoposide had been chosen regularly. Moreover, it is known that SCLC patients have a risk of developing brain metastases. This risk can be reduced by prophylactic cranial irradiation ${ }^{22}$ that was performed only in some of our patients for unknown reasons with potential adverse impact on survival.

In conclusion, this study demonstrated that multimodality treatment including surgery could be performed safely and led to considerable survival in stage IA-IIIB SCLC. R0 resection was the only statistically significant factor extending survival. It could be achieved in most patients and was associated with a low risk of locoregional relapse. Because surgery can enable R0 resection, considering stage IA-IIIB SCLC patients for multimodality therapy including surgery seems reasonable if $\mathrm{R} 0$ resection is expected to be obtainable. Nevertheless, its definitive significance should be evaluated in prospective controlled randomized studies comparing multimodality treatment including surgery to chemoradiotherapy. Standardization should be mandatory for surgical techniques, as well as chemotherapy, thoracic radiotherapy, and prophylactic cranial irradiation. This would help obtain reproducible results and determine best practice for managing stage IA-IIIB SCLC.

Note

The study protocol had approval by the local ethics committee (www.laekh.de, study identification number FF 139/ 2016).

\section{References}

1 Turrisi AT III, Kim K, Blum R, et al. Twice-daily compared with once-daily thoracic radiotherapy in limited small-cell lung cancer treated concurrently with cisplatin and etoposide. N Engl J Med 1999;340(04):265-271 
2 Lad T, Piantadosi S, Thomas P, Payne D, Ruckdeschel J, Giaccone G. A prospective randomized trial to determine the benefit of surgical resection of residual disease following response of small cell lung cancer to combination chemotherapy. Chest 1994;106(6, Suppl):320S-323S

3 Badzio A, Kurowski K, Karnicka-Mlodkowska H, Jassem J. A retrospective comparative study of surgery followed by chemotherapy vs. non-surgical management in limited-disease small cell lung cancer. Eur J Cardiothorac Surg 2004;26(01):183-188

4 Eberhardt W, Stamatis G, Stuschke M, et al. Prognostically orientated multimodality treatment including surgery for selected patients of small-cell lung cancer patients stages IB to IIIB: long-term results of a phase II trial. Br J Cancer 1999;81 (07):1206-1212

5 Zhang J, Li S, Chen X, et al. Retrospective study of surgery versus non-surgical management in limited-disease small cell lung cancer. Thorac Cancer 2014;5(05):405-410

6 Wakeam E, Acuna SA, Leighl NB, et al. Surgery versus chemotherapy for early and locally advanced small-cell lung cancer: a propensity matched analysis of survival. Lung Cancer 2017; 109:78-88

7 Shepherd FA, Ginsberg RJ, Feld R, Evans WK, Johansen E. Surgical treatment for limited small-cell lung cancer. The University of Toronto Lung Oncology Group experience. J Thorac Cardiovasc Surg 1991;101(03):385-393

8 Welter S, Aigner C, Roesel C. The role of surgery in high grade neuroendocrine tumours of the lung. J Thorac Dis 2017;9 (Suppl 15):S1474-S1483

9 Schreiber D, Rineer J, Weedon J, et al. Survival outcomes with the use of surgery in limited-stage small cell lung cancer: should its role be re-evaluated? Cancer 2010;116(05):1350-1357

10 Sobin LH, Gospodarowicz MK, Wittekind C. TNM Classification of Malignant Tumours. 7th ed. Oxford: Wiley-Blackwell; 2009

11 Bölükbas S, Eberlein M, Schirren J. Role of mediastinal lymph node dissection in non-small cell lung cancer. Front Radiat Ther Oncol 2010;42:78-86
12 Lim E, Belcher E, Yap YK, Nicholson AG, Goldstraw P. The role of surgery in the treatment of limited disease small cell lung cancer: time to reevaluate. J Thorac Oncol 2008;3(11):1267-1271

13 Fukuoka M, Furuse K, Saijo N, et al. Randomized trial of cyclophosphamide, doxorubicin, and vincristine versus cisplatin and etoposide versus alternation of these regimens in small-cell lung cancer. J Natl Cancer Inst 1991;83(12):855-861

14 Bülzebruck H, Krysa S, Bauer E, Probst G, Drings P, Vogt-Moykopf I. Validation of the TNM classification (4th edn) for lung cancer: first results of a prospective study of 1086 patients with surgical treatment. Eur J Cardiothorac Surg 1991;5(07):356-362

15 Yang CJ, Chan DY, Speicher PJ, et al. Surgery versus optimal medical management for N1 small cell lung cancer. Ann Thorac Surg 2017;103(06):1767-1772

$16 \mathrm{Xu} \mathrm{YJ}$, Zheng $\mathrm{H}$, Gao $\mathrm{W}$, et al. Is neoadjuvant chemotherapy mandatory for limited-disease small-cell lung cancer? Interact Cardiovasc Thorac Surg 2014;19(06):887-893

17 Shepherd FA, Evans WK, Feld R, et al. Adjuvant chemotherapy following surgical resection for small-cell carcinoma of the lung. J Clin Oncol 1988;6(05):832-838

18 Wong AT, Rineer J, Schwartz D, Schreiber D. Assessing the impact of postoperative radiation therapy for completely resected limited-stage small cell lung cancer using the National Cancer Database. J Thorac Oncol 2016;11(02):242-248

19 Wang HJ, Sun KL, Zhang XR, Sun Y, Shi YK. Combined modality therapy for small cell lung cancer patient with limited stage disease in Chinese]. Zhonghua Zhong Liu Za Zhi 2007;29(09):701-703

20 Kawano D, Okamoto T, Fujishita T, et al. Surgical results of resectable small cell lung cancer. Thorac Cancer 2015;6(02):141-145

21 Yuequan J, Zhi Z, Chenmin X. Surgical resection for small cell lung cancer: pneumonectomy versus lobectomy. ISRN Surg 2012; 2012:101024

22 Aupérin A, Arriagada R, Pignon JP, et al; Prophylactic Cranial Irradiation Overview Collaborative Group. Prophylactic cranial irradiation for patients with small-cell lung cancer in complete remission. N Engl J Med 1999;341(07):476-484 